\title{
Regularity and time-periodicity for a nematic liquid crystal model
}

\author{
Blanca Climent-Ezquerra*, Francisco Guillén-González*, \\ M. Jesus Moreno-Iraberte
}

\author{
Dpto. Ecuaciones Diferenciales y Análisis Numérico, Universidad de Sevilla, \\ Aptdo. 1160, 41080 Sevilla, Spain. \\ E-mails: bcliment@us.es, guillen@us.es, mjmoreno@us.es
}

\begin{abstract}
In this paper two main results are obtained for a nematic liquid crystal model with timedependent boundary Dirichlet data for the orientation of the crystal molecules. First, the initial-boundary problem is considered, obtaining the existence of global in time (up to infinity time) weak solution, the existence of global regular solution for viscosity coefficient big enough, and the weak/strong uniqueness. Second, using these previous results and the existence of time-periodic weak solutions proved in [2], the regularity of any time-periodic weak solution is deduced for viscosity coefficient big enough.
\end{abstract}

Keywords: solution up to infinity time, time-periodic solutions, uniqueness, Navier-Stokes equations, Nematic liquid crystal models, coupled non-linear parabolic system.

\section{Introduction}

In this work, a simplified Ericksen-Leslie version for a nematic liquid crystal model is considered; see for instance [8] for a formulation of a more complete liquid crystal problem.

This model can be seen as a variant of the Navier-Stokes problem (respect to the velocitypressure unknowns $(\boldsymbol{u}, p)$ ) coupled with a convection-diffusion system for a new variable $\boldsymbol{d}$, which is a unit vectorial function modelling the orientation of the crystal molecules. On the other hand, it is usual to consider an approximation by Ginzburg-Landau penalization ([1]) for the constraint $|\boldsymbol{d}|=1(|\boldsymbol{d}|=|\boldsymbol{d}(t, x)|$ denotes the point-wise euclidean norm). This penalized model (where the constraint $|\boldsymbol{d}|=1$ is relaxed by $|\boldsymbol{d}| \leq 1$ ) was introduced by Lin in [6] and studied (from a mathematical point of view) by Lin and Liu in [7, 8]. Coutand and Shkoller in [4] also studied this simplified model but including stretching effects.

We assume a (newtonian) fluid confined in an open bounded domain $\Omega \subset \mathbb{R}^{N}(N=2$ or 3 ) with regular boundary $\partial \Omega$. In the penalized model the constraint $|\boldsymbol{d}|=1$ is partially conserved to

*First and second authors have been partially financed by the projets P06-FQM-02373 and MTM2006-07932. 
$|\boldsymbol{d}| \leq 1$ as consequence of the maximum principle for the Ginzburg-Landau equation considering the function $\boldsymbol{f}(\boldsymbol{d})=\frac{1}{\varepsilon^{2}}\left(|\boldsymbol{d}|^{2}-1\right) \boldsymbol{d}$ where $\varepsilon>0$ is the penalization parameter. There exists a potential function $\boldsymbol{F}(\boldsymbol{d})=\frac{1}{4 \varepsilon^{2}}\left(|\boldsymbol{d}|^{2}-1\right)^{2}$ such that $\boldsymbol{f}(\boldsymbol{d})=\nabla_{\boldsymbol{d}} \boldsymbol{F}(\boldsymbol{d})$ for each $\boldsymbol{d} \in \mathbb{R}^{N}$. Then, we consider the following PDE system in $(0,+\infty) \times \Omega$ :

$$
\left\{\begin{array}{l}
\partial_{t} \boldsymbol{u}+(\boldsymbol{u} \cdot \nabla) \boldsymbol{u}-\nu \Delta \boldsymbol{u}+\nabla p=-\nabla \boldsymbol{d}^{t} \Delta \boldsymbol{d}, \quad \nabla \cdot \boldsymbol{u}=0 \\
\partial_{t} \boldsymbol{d}+(\boldsymbol{u} \cdot \nabla) \boldsymbol{d}=\Delta \boldsymbol{d}-\boldsymbol{f}(\boldsymbol{d}), \quad|\boldsymbol{d}| \leq 1
\end{array}\right.
$$

The constants $\nu, \lambda$ and $\gamma$ are positive, representing respectively, the fluid viscosity, an elasticity constant and a relaxation time (for simplicity we consider $\lambda=\gamma=1$ and $\nu>0$ and for the last result, large enough). The problem (1) is completed with the (Dirichlet) boundary conditions

$$
\boldsymbol{u}(x, t)=0, \quad \boldsymbol{d}(x, t)=\boldsymbol{h}(x, t) \quad \text { on } \partial \Omega \times(0,+\infty)
$$

(assuming as in [2] a time-depending boundary data for $\boldsymbol{d}$ given by $\boldsymbol{h}: \partial \Omega \times(0,+\infty) \mapsto \mathbb{R}^{N} ;$ in $[7,8]$ only a time-independent boundary data is considered) and either the initial condition

$$
\boldsymbol{u}(x, 0)=\boldsymbol{u}_{0} \quad \boldsymbol{d}(x, 0)=\boldsymbol{d}_{0} \quad \text { in } \Omega
$$

or the time-periodic condition:

$$
\boldsymbol{u}(x, 0)=\boldsymbol{u}(x, T), \quad \boldsymbol{d}(x, 0)=\boldsymbol{d}(x, T) \quad \text { in } \Omega,
$$

where $T>0$ is a given final time. In this last case, we assume, moreover, that $\boldsymbol{h}(0)=\boldsymbol{h}(T)$.

This model has, beside well known difficulties for the Navier-Stokes problem (a nonlinear parabolic system with the free divergence constraint related to the pressure), other different difficulties which come from the strongly nonlinear coupling between the orientation vector $\boldsymbol{d}$ and the velocity-pressure $(\boldsymbol{u}, p)$ and from the constraint $|\boldsymbol{d}| \leq 1$.

An essential characteristic of the problem for $\boldsymbol{d}$ (given $\boldsymbol{u}$ ), either the initial-value problem with (3) or the time-periodic case with (4), is the following weak maximum principle (see [7, 2]): Assume $|\boldsymbol{h}| \leq 1$ on $\partial \Omega \times(0, T)$ and either $\left|\boldsymbol{d}_{0}\right| \leq 1$ in $\Omega$ for the initial-value problem or $\boldsymbol{h}(0)=\boldsymbol{h}(T)$ on $\partial \Omega$ for the time-periodic problem. Then, given $\boldsymbol{u} \in L^{2}(0, T ; \boldsymbol{V}) \cap L^{\infty}(0, T ; \boldsymbol{H})$ (see the notations below for the definition of spaces $\boldsymbol{V}$ and $\boldsymbol{H}$ ), any point-wise solution for the $\boldsymbol{d}$-problem verifies $|\boldsymbol{d}(x, t)| \leq 1$ a.e. in $\Omega \times(0, T)$.

In [7], considering the initial-value problem (1)-(3) with time-independent boundary conditions for $\boldsymbol{d}$, authors prove existence of global weak solution (with $\boldsymbol{u} \in L^{\infty}\left(\boldsymbol{L}^{2}\right) \cap L^{2}\left(\boldsymbol{H}^{1}\right), \boldsymbol{d} \in L^{\infty}\left(\boldsymbol{H}^{1}\right) \cap$ $L^{2}\left(\boldsymbol{H}^{2}\right)$ ), existence of global regular solution (with $\boldsymbol{u} \in L^{\infty}\left(\boldsymbol{H}^{1}\right) \cap L^{2}\left(\boldsymbol{H}^{2}\right), \boldsymbol{d} \in L^{\infty}\left(\boldsymbol{H}^{2}\right) \cap L^{2}\left(\boldsymbol{H}^{3}\right)$ ) if $\nu$ is big enough for $N=3$ and uniqueness of regular solutions. However, in these previous results of [7] there is an important simplification; the boundary data $\boldsymbol{h}$ does not depend on time. In this case, the time-periodic problem $(1),(2),(4)$ with boundary condition independent of the time $\left(\boldsymbol{d}(x, t)_{\mid \partial \Omega \times(0, T)}=\boldsymbol{d}_{0}(x)\right)$, leads to a trivial problem (see [2]), because all "static" solutions $\boldsymbol{u}=0$ and $\boldsymbol{d}$ verifying stationary problem $-\Delta \boldsymbol{d}+\boldsymbol{f}(\boldsymbol{d})=0$ in $\Omega, \boldsymbol{d}_{\mid \partial \Omega}=\boldsymbol{d}_{0}$, are in particular time-periodic solutions. Respect to the nontrivial case of time-dependent boundary condition, the existence of weak time-periodic solutions of $(1),(2),(4)$ is proved in $[2]$. 
The main results of the present article are the following: always for boundary data $\boldsymbol{h}$ depending on the time, we prove existence of global weak solution (defined in $[0,+\infty)$ ) for the initial value problem (1)-(3), existence of global strong solutions under the constraint of viscosity coefficient $\nu$ big enough and uniqueness of strong/weak solutions, that is any weak solution coincides with the strong solution (if this strong solution exists). Moreover, we prove existence of regular timeperiodic solutions under the same type of constraint.

A existence result of regular time-periodic solutions for a generalized Boussinesq model can be seen in [3].

The paper is organized as follows. In Section 2, some differential inequalities in weak norms are deduced, whereas Section 3 is devoted to obtain differential inequalities in strong norms. In Section 4, the global in time solution of the initial-value problem is studied (at infinity time), and finally the existence of strong time-periodic solution is obtained in Section 5, using results of Section 4 and the existence of weak time-periodic solution of [2].

\section{Notations}

- We denote $Q=(0,+\infty) \times \Omega, Q_{T}=(0, T) \times \Omega, \Sigma=(0,+\infty) \times \partial \Omega$ and $\Sigma_{T}=(0, T) \times \partial \Omega$.

- In general, the notation will be abridged. We set $L^{p}=L^{p}(\Omega), p \geq 1, H_{0}^{1}=H_{0}^{1}(\Omega)$, etc. If $X=X(\Omega)$ is a space of functions defined in the open set $\Omega$, we denote by $L^{p}(X)$ the Banach space $L^{p}(0, T ; X)$. Also, boldface letters will be used for vectorial spaces, for instance $\mathbf{L}^{2}=L^{2}(\Omega)^{N}$.

- The $L^{p}$ norm is denoted by $|\cdot|_{p}, 1 \leq p \leq \infty$, the $H^{m}$ norm by $\|\cdot\|_{m}$ (in particular $|\cdot|_{2}=\|\cdot\|_{0}$ ) and the product norm in $H^{n} \times H^{m}$ by $\|\cdot\|_{m \times n}$. The inner product of $L^{2}(\Omega)$ is denoted by $(\cdot, \cdot)$.

- We set $\mathcal{V}$ the space formed by all fields $\boldsymbol{u} \in C_{0}^{\infty}(\Omega)^{N}$ satisfying $\nabla \cdot \boldsymbol{u}=0$. We denote $\boldsymbol{H}$ (respectively $\boldsymbol{V}$ ) the closure of $\mathcal{V}$ in $\boldsymbol{L}^{2}$ (respectively $\boldsymbol{H}^{1}$ ). $\boldsymbol{H}$ and $\boldsymbol{V}$ are Hilbert spaces for the norms $|\cdot|_{2}$ and $\|\cdot\|_{1}$, respectively. Furthermore,

$$
\boldsymbol{H}=\left\{\boldsymbol{u} \in \boldsymbol{L}^{2} ; \nabla \cdot \boldsymbol{u}=0, \boldsymbol{u} \cdot \mathbf{n}=0 \text { on } \partial \Omega\right\}, \quad \boldsymbol{V}=\left\{\boldsymbol{u} \in \boldsymbol{H}^{1} ; \nabla \cdot \boldsymbol{u}=0, \boldsymbol{u}=0 \text { on } \partial \Omega\right\}
$$

- In the sequel, $C, D>0$ will denote different constants, depending only on the fixed data of the problem, as $\Omega, \lambda, \gamma$.

\section{Differential inequalities in weak norms}

\subsection{A lifting function}

We define $\tilde{\boldsymbol{d}}(t)$ as the weak solution of the Laplace-Dirichlet problem

$$
\begin{cases}-\Delta \widetilde{\boldsymbol{d}}=0 & \text { in } \Omega, \\ \widetilde{\boldsymbol{d}}=\boldsymbol{h}(t) & \text { on } \partial \Omega .\end{cases}
$$


In the time-periodic case, since by hypothesis $\boldsymbol{h}(0)=\boldsymbol{h}(T)$ on $\partial \Omega$, then $\widetilde{\boldsymbol{d}}(0)=\widetilde{\boldsymbol{d}}(T)$ in $\Omega$.

Therefore, if we define $\widehat{\boldsymbol{d}}(t)=\boldsymbol{d}(t)-\widetilde{\boldsymbol{d}}(t)$, then $\Delta \widehat{\boldsymbol{d}}=\Delta \boldsymbol{d}$ in $\Omega \times(0, T)$ and $\widehat{\boldsymbol{d}}=0$ on $\partial \Omega \times(0, T)$. In the time-periodic case, $\boldsymbol{d}(0)=\boldsymbol{d}(T)$ if and only if $\widehat{\boldsymbol{d}}(0)=\widehat{\boldsymbol{d}}(T)$. Then, we can rewrite the problem (1)-(2) in the variables $(\boldsymbol{u}, \widehat{\boldsymbol{d}})$ (with $\boldsymbol{d}=\widehat{\boldsymbol{d}}+\widetilde{\boldsymbol{d}}$ ) as follows:

$$
\left\{\begin{array}{l}
\partial_{t} \boldsymbol{u}+(\boldsymbol{u} \cdot \nabla) \boldsymbol{u}-\nu \Delta \boldsymbol{u}+\nabla p+\nabla \boldsymbol{d}^{t} \Delta \widehat{\boldsymbol{d}}=0, \quad \nabla \cdot \boldsymbol{u}=0 \quad \text { in } Q_{T} \\
\partial_{t} \widehat{\boldsymbol{d}}+(\boldsymbol{u} \cdot \nabla) \boldsymbol{d}-\Delta \widehat{\boldsymbol{d}}+\boldsymbol{f}(\boldsymbol{d})=-\partial_{t} \widetilde{\boldsymbol{d}} \quad \text { in } Q_{T} \\
\boldsymbol{u}=0, \quad \widehat{\boldsymbol{d}}=0 \quad \text { on } \partial \Omega \times(0, T)
\end{array}\right.
$$

jointly with either the initial condition $\boldsymbol{u}(0)=\boldsymbol{u}_{0}, \widehat{\boldsymbol{d}}(0)=\boldsymbol{d}_{0}-\widetilde{\boldsymbol{d}}(0)$ or the time-periodic conditions $\boldsymbol{u}(0)=\boldsymbol{u}(T), \widehat{\boldsymbol{d}}(0)=\widehat{\boldsymbol{d}}(T)$.

Remark: The choice of this type of lifting function allows us to obtain estimates up to infinity time, which is not possible with the lifting function that we will consider in Section 3.

\subsection{Differential inequalities}

We will give two different differential equalities in the next two lemmas.

Lemma 1 If $\boldsymbol{u}$ and $\boldsymbol{d}$ are regular enough, the following differential inequality holds:

$$
\frac{d}{d t}\left(|\boldsymbol{u}|_{2}^{2}+|\nabla \widehat{\boldsymbol{d}}|_{2}^{2}\right)+2 \nu|\nabla \boldsymbol{u}|_{2}^{2}+|\Delta \widehat{\boldsymbol{d}}|_{2}^{2} \leq 2\left(|\boldsymbol{f}(\boldsymbol{d})|_{2}^{2}+\left|\partial_{t} \widetilde{\boldsymbol{d}}\right|_{2}^{2}\right),
$$

Proof: Taking $\boldsymbol{u}$ and $-\boldsymbol{\Delta} \widehat{\boldsymbol{d}}$ as test functions in (6), adding up, taking into account that

$$
((\boldsymbol{u} \cdot \nabla) \boldsymbol{u}, \boldsymbol{u})=0 \quad \text { and } \quad\left(\nabla \boldsymbol{d}^{t} \Delta \widehat{\boldsymbol{d}}, \boldsymbol{u}\right)-((\boldsymbol{u} \cdot \nabla) \boldsymbol{d}, \Delta \widehat{\boldsymbol{d}})=0
$$

one arrives (at least formally) at the following energy equality:

$$
\frac{1}{2} \frac{d}{d t}\left(|\boldsymbol{u}|_{2}^{2}+|\nabla \widehat{\boldsymbol{d}}|_{2}^{2}\right)+\nu|\nabla \boldsymbol{u}|_{2}^{2}+|\Delta \widehat{\boldsymbol{d}}|_{2}^{2}=(\boldsymbol{f}(\boldsymbol{d}), \Delta \widehat{\boldsymbol{d}})+\left(\partial_{t} \widetilde{\boldsymbol{d}}, \Delta \widehat{\boldsymbol{d}}\right)
$$

Consequently, applying Young inequality, one has (7).

Lemma 2 If $\boldsymbol{u}$ and $\boldsymbol{d}$ are regular enough, the following differential inequality holds:

$$
\frac{d}{d t}\left(|\boldsymbol{u}|_{2}^{2}+|\nabla \widehat{\boldsymbol{d}}|_{2}^{2}+2 \int_{\Omega} F(\boldsymbol{d})\right)+2 \nu|\nabla \boldsymbol{u}|_{2}^{2}+|\Delta \widehat{\boldsymbol{d}}-\boldsymbol{f}(\boldsymbol{d})|_{2}^{2} \leq\left|\partial_{t} \widetilde{\boldsymbol{d}}\right|_{2}^{2}
$$

Proof: Taking $\boldsymbol{u}$ and $-\Delta \widehat{\boldsymbol{d}}+\boldsymbol{f}(\boldsymbol{d})$ as test functions in (6), adding up and taking into account (8), $\partial_{t} \boldsymbol{d} \cdot \boldsymbol{f}(\boldsymbol{d})=\partial_{t} \boldsymbol{F}(\boldsymbol{d})$ and $((\boldsymbol{u} \cdot \nabla) \boldsymbol{d}, \boldsymbol{f}(\boldsymbol{d}))=0$, one obtains

$$
\frac{1}{2} \frac{d}{d t}\left(|\boldsymbol{u}|_{2}^{2}+|\nabla \widehat{\boldsymbol{d}}|_{2}^{2}+2 \int_{\Omega} F(\boldsymbol{d})\right)+\nu|\nabla \boldsymbol{u}|_{2}^{2}+|\Delta \widehat{\boldsymbol{d}}-\boldsymbol{f}(\boldsymbol{d})|_{2}^{2}=\left(\partial_{t} \widetilde{\boldsymbol{d}}, \Delta \widehat{\boldsymbol{d}}-\boldsymbol{f}(\boldsymbol{d})\right) .
$$

$* * * * * * * * * * * * * * * * * *$ By rewriting the second term as

$$
\left(\partial_{t} \widetilde{\boldsymbol{d}}, \Delta \widehat{\boldsymbol{d}}\right)=\left(\partial_{t} \widetilde{\boldsymbol{d}}, \Delta \widehat{\boldsymbol{d}}-\boldsymbol{f}(\boldsymbol{d})\right)+\left(\partial_{t} \widetilde{\boldsymbol{d}}, \boldsymbol{f}(\boldsymbol{d})\right),
$$

by using $|\boldsymbol{f}(\boldsymbol{d})| \leq \frac{1}{\varepsilon^{2}}$ owing to the maximum principle $|\boldsymbol{d}| \leq 1$ one has

$$
\left|\left(\partial_{t} \widetilde{\boldsymbol{d}}, \Delta \widehat{\boldsymbol{d}}\right)\right| \leq \frac{1}{2}\left|\partial_{t} \widetilde{\boldsymbol{d}}\right|_{2}^{2}+\frac{1}{2}|\Delta \widehat{\boldsymbol{d}}-\boldsymbol{f}(\boldsymbol{d})|_{2}^{2}+\frac{1}{\varepsilon^{2}}\left|\partial_{t} \widetilde{\boldsymbol{d}}\right|_{1} .
$$

Therefore, one arrives (at least formally) at (9). 


\section{Differential inequalities in regular norms}

\subsection{A lifting function}

We will consider another suitable lifting function $\tilde{\boldsymbol{d}}$ for the boundary data $\boldsymbol{h}$ (that we denote equal) in such a way that we could made estimates of $\boldsymbol{H}^{3}$-type for the homogeneous variable related to $\boldsymbol{d}$ (see [5]). Concretely, we define $\widetilde{\boldsymbol{d}}$ as the solution of the problem:

$$
\left\{\begin{array}{l}
\partial_{t} \widetilde{\boldsymbol{d}}-\Delta \widetilde{\boldsymbol{d}}=0 \quad \text { in } Q_{T} \\
\widetilde{\boldsymbol{d}}=\boldsymbol{h} \quad \text { on } \partial \Omega \times(0, T)
\end{array}\right.
$$

jointly $\tilde{\boldsymbol{d}}(0)=\boldsymbol{d}_{0}$ in $\Omega$ for the initial valued problem or $\tilde{\boldsymbol{d}}(0)=\tilde{\boldsymbol{d}}(T)$ in $\Omega$ for the time-periodic case.

Then, the $\boldsymbol{d}$-problem of (1) can be rewritten as follows:

$$
\left\{\begin{array}{l}
\partial_{t} \widehat{\boldsymbol{d}}+(\boldsymbol{u} \cdot \nabla) \boldsymbol{d}-\Delta \widehat{\boldsymbol{d}}+\boldsymbol{f}(\boldsymbol{d})=0 \quad \text { in } Q_{T}, \\
\widehat{\boldsymbol{d}}=0 \quad \text { on } \partial \Omega \times(0, T)
\end{array}\right.
$$

and $\widehat{\boldsymbol{d}}(0)=0$ in $\Omega$ for the initial-valued problem or $\widehat{\boldsymbol{d}}(0)=\widehat{\boldsymbol{d}}(T)$ in $\Omega$ for the time-periodic case. As consequence of the maximum principle $|\boldsymbol{d}| \leq 1$ and $|\widetilde{\boldsymbol{d}}| \leq 1$. Although we do not know if $|\widehat{\boldsymbol{d}}| \leq 1$, we have $\|\widehat{\boldsymbol{d}}\|_{L^{\infty}\left(L^{\infty}\right)} \leq\|\boldsymbol{d}\|_{L^{\infty}\left(L^{\infty}\right)}+\|\widetilde{\boldsymbol{d}}\|_{L^{\infty}\left(L^{\infty}\right)} \leq 2$.

We are going to consider the following equivalents norms:

$$
\begin{gathered}
\|\boldsymbol{u}\|_{1} \approx|\nabla \boldsymbol{u}|_{2}, \quad\|\widehat{\boldsymbol{d}}\|_{1} \approx|\nabla \widehat{\boldsymbol{d}}|_{2} \quad \text { in } \boldsymbol{H}_{0}^{1}, \\
\|\boldsymbol{u}\|_{2} \approx|\Delta \boldsymbol{u}|_{2}, \quad\|\widehat{\boldsymbol{d}}\|_{2} \approx|\Delta \widehat{\boldsymbol{d}}|_{2} \quad \text { in } \boldsymbol{H}_{0}^{1} \cap \boldsymbol{H}^{2} \\
\|\widehat{\boldsymbol{d}}\|_{3} \approx|\nabla(\Delta \widehat{\boldsymbol{d}})|_{2}+|\Delta \widehat{\boldsymbol{d}}|_{2}=\|\Delta \widehat{\boldsymbol{d}}\|_{1} \quad \text { in } \boldsymbol{H}_{0}^{1} \cap \boldsymbol{H}^{3}
\end{gathered}
$$

Remark: Owing to the lifting function considered in this section, we have that $\Delta \widehat{\boldsymbol{d}}-\left.\boldsymbol{f}(\boldsymbol{d})\right|_{\partial \Omega}=0$ because the rest of the terms in (11) vanish on the boundary. That is not true with the lifting function of the Section 2.

\subsection{Differential inequalities}

In the previous conditions, the following regularity result will be frequently used.

Lemma 3 Assume $\boldsymbol{d}=\widehat{\boldsymbol{d}}+\widetilde{\boldsymbol{d}}$, with $|\boldsymbol{d}| \leq 1$,

a) if $\Delta \widehat{\boldsymbol{d}}-\boldsymbol{f}(\boldsymbol{d}) \in \boldsymbol{L}^{2}(\Omega)$ and $\widetilde{\boldsymbol{d}} \in \boldsymbol{H}^{2}(\Omega)$, then $\boldsymbol{d} \in \boldsymbol{H}^{2}(\Omega)$ and

$$
\|\boldsymbol{d}\|_{2} \leq\|\widetilde{\boldsymbol{d}}\|_{2}+C\left(1+|\Delta \widehat{\boldsymbol{d}}-\boldsymbol{f}(\boldsymbol{d})|_{2}\right),
$$

b) if $\Delta \widehat{\boldsymbol{d}}-\boldsymbol{f}(\boldsymbol{d}) \in \boldsymbol{H}^{1}(\Omega)$ and $\widetilde{\boldsymbol{d}} \in \boldsymbol{H}^{3}(\Omega)$, then $\boldsymbol{d} \in \boldsymbol{H}^{3}(\Omega)$ and

$$
\|\boldsymbol{d}\|_{3} \leq\|\widetilde{\boldsymbol{d}}\|_{3}+C\left(|\nabla \boldsymbol{d}|_{2}+|\nabla(\Delta \widehat{\boldsymbol{d}}-\boldsymbol{f}(\boldsymbol{d}))|_{2}\right) .
$$


Proof: For the proof, it is fundamental that $\Delta \widehat{\boldsymbol{d}}-\boldsymbol{f}(\boldsymbol{d})=0$ on $\partial \Omega$. We have $\|\boldsymbol{d}\|_{2} \leq\|\widehat{\boldsymbol{d}}\|_{2}+\|\tilde{\boldsymbol{d}}\|_{2}$ and $\|\boldsymbol{d}\|_{3} \leq\|\widehat{\boldsymbol{d}}\|_{3}+\|\widetilde{\boldsymbol{d}}\|_{3}$. Then, by adding and subtracting $\boldsymbol{f}(\boldsymbol{d})$ into the norms $\|\widehat{\boldsymbol{d}}\|_{2} \approx|\Delta \widehat{\boldsymbol{d}}|_{2}$ and $\|\widehat{\boldsymbol{d}}\|_{3} \approx\|\Delta \widehat{\boldsymbol{d}}\|_{1}$, we obtain the first and second inequality, respectively, using that $|\boldsymbol{f}(\boldsymbol{d})| \leq C$ and $|\nabla \boldsymbol{f}(\boldsymbol{d})| \leq C|\nabla \boldsymbol{d}|$.

Lemma 4 Assume $\tilde{\boldsymbol{d}} \in L^{\infty}\left(0,+\infty ; \boldsymbol{H}^{3}(\Omega)\right), \boldsymbol{u} \in L^{\infty}\left(0,+\infty ; \boldsymbol{L}^{2}(\Omega)\right)$ and $\boldsymbol{d} \in L^{\infty}\left(0,+\infty ; \boldsymbol{H}^{1}(\Omega)\right)$. Then, if $\boldsymbol{u}$ and $\boldsymbol{d}$ are regular enough, the following differential inequality holds:

$$
\begin{aligned}
& \frac{d}{d t}\left(\|\boldsymbol{u}\|_{1}^{2}+|\Delta \widehat{\boldsymbol{d}}-\boldsymbol{f}(\boldsymbol{d})|_{2}^{2}\right)+\nu\|\boldsymbol{u}\|_{2}^{2}+2|\nabla(\Delta \widehat{\boldsymbol{d}}-\boldsymbol{f}(\boldsymbol{d}))|_{2}^{2} \\
& \leq D\left(1+|\Delta \widehat{\boldsymbol{d}}-\boldsymbol{f}(\boldsymbol{d})|_{2}^{2}\right)+\frac{E}{\nu}\left(\|\boldsymbol{u}\|_{1}\|\boldsymbol{u}\|_{2}^{2}+\left(1+|\Delta \widehat{\boldsymbol{d}}-\boldsymbol{f}(\boldsymbol{d})|_{2}^{2}\right)|\nabla(\Delta \widehat{\boldsymbol{d}}-\boldsymbol{f}(\boldsymbol{d}))|_{2}^{2}\right),
\end{aligned}
$$

where $D, E>0$ are constants independent of $\nu$.

Proof: Taking $A \boldsymbol{u}$ as test functions in the $\boldsymbol{u}$-system of (1) ( $A$ being the Stokes operator) and applying adequately Hölder and Young's inequalities, one obtains:

$$
\begin{aligned}
& \frac{d}{d t}\|\boldsymbol{u}\|_{1}^{2}+\frac{4}{3} \nu\|\boldsymbol{u}\|_{2}^{2} \leq \frac{C}{\nu}\left(|(\boldsymbol{u} \cdot \nabla) \boldsymbol{u}|_{2}^{2}+\left|\nabla^{t} \boldsymbol{d} \Delta \boldsymbol{d}\right|_{2}^{2}\right) \\
& \leq \frac{C}{\nu}\left(|\boldsymbol{u}|_{3}^{2}|\nabla \boldsymbol{u}|_{6}^{2}+\left|\nabla^{t} \boldsymbol{d}\right|_{3}^{2}|\Delta \boldsymbol{d}|_{6}^{2}\right) \leq \frac{C}{\nu}\left(|\boldsymbol{u}|_{2}\|\boldsymbol{u}\|_{1}\|\boldsymbol{u}\|_{2}^{2}+\|\boldsymbol{d}\|_{1}\|\boldsymbol{d}\|_{2}\|\boldsymbol{d}\|_{3}^{2}\right)
\end{aligned}
$$

Hence, owing to Lemma 3 , weak estimates $\left(|\boldsymbol{u}(t)|_{2} \leq C,\|\boldsymbol{d}(t)\|_{1} \leq C\right.$ a.e. $\left.t \in(0,+\infty)\right)$ and strong regularity of $\widetilde{\boldsymbol{d}} \in L^{\infty}\left(0,+\infty ; \boldsymbol{H}^{3}(\Omega)\right)$ (in particular, inequalities of Lemma 3 derive in the simplest inequalities: $\|\boldsymbol{d}\|_{2} \leq C\left(1+|\Delta \widehat{\boldsymbol{d}}-\boldsymbol{f}(\boldsymbol{d})|_{2}\right)$ and $\left.\|\boldsymbol{d}\|_{3} \leq C\left(1+|\nabla(\Delta \widehat{\boldsymbol{d}}-\boldsymbol{f}(\boldsymbol{d}))|_{2}\right)\right)$, we have:

$$
\begin{aligned}
\frac{d}{d t}\|\boldsymbol{u}\|_{1}^{2}+\frac{4}{3} \nu\|\boldsymbol{u}\|_{2}^{2} & \leq \frac{C}{\nu}\left(\|\boldsymbol{u}\|_{1}\|\mathbf{u}\|_{2}^{2}+\left(1+|\Delta \widehat{\boldsymbol{d}}-\boldsymbol{f}(\boldsymbol{d})|_{2}\right)|\nabla(\Delta \widehat{\boldsymbol{d}}-\boldsymbol{f}(\boldsymbol{d}))|_{2}^{2}\right) \\
& +C\left(1+|\Delta \widehat{\boldsymbol{d}}-\boldsymbol{f}(\boldsymbol{d})|_{2}\right) .
\end{aligned}
$$

In the last term we have considered that $C / \nu$ is uniformly bounded respect to $\nu$, as $\nu \geq \nu_{0}$.

By taking gradient in the $\widehat{\boldsymbol{d}}$-system (11), multiplying by $-\nabla(\Delta \widehat{\boldsymbol{d}}-\boldsymbol{f}(\boldsymbol{d}))$, integrating by parts in the $\partial_{t} \widehat{\boldsymbol{d}}$-term (where all the boundary terms vanish owing to the choice of the lifting function $\boldsymbol{d}$ that implies $\left.\left.(\Delta \widehat{\boldsymbol{d}}-\boldsymbol{f}(\boldsymbol{d}))\right|_{\partial \Omega}=0\right)$ and adding both sides the term $-\left(\partial_{t} \boldsymbol{f}(\boldsymbol{d}), \Delta \widehat{\boldsymbol{d}}-\boldsymbol{f}(\boldsymbol{d})\right)$, we find:

$$
\begin{gathered}
\frac{1}{2} \frac{d}{d t}|\Delta \widehat{\boldsymbol{d}}-\boldsymbol{f}(\boldsymbol{d})|_{2}^{2}+|\nabla(\Delta \widehat{\boldsymbol{d}}-\boldsymbol{f}(\boldsymbol{d}))|_{2}^{2}=-\left(\partial_{t} \boldsymbol{f}(\boldsymbol{d}), \Delta \widehat{\boldsymbol{d}}-\boldsymbol{f}(\boldsymbol{d})\right) \\
-((\nabla \boldsymbol{u} \cdot \nabla) \boldsymbol{d}, \nabla(\Delta \widehat{\boldsymbol{d}}-\boldsymbol{f}(\boldsymbol{d})))+(\boldsymbol{u} \cdot \nabla \nabla \mathbf{d}, \nabla(\Delta \widehat{\boldsymbol{d}}-\boldsymbol{f}(\boldsymbol{d})))
\end{gathered}
$$

By using the $\widehat{\boldsymbol{d}}$-system we have that

$$
-\partial_{t} \boldsymbol{f}(\boldsymbol{d})=-\nabla_{d} \boldsymbol{f}(\boldsymbol{d}) \partial_{t} \boldsymbol{d}=\nabla_{d} \boldsymbol{f}(\boldsymbol{d})((\boldsymbol{u} \cdot \nabla) \boldsymbol{d}-\Delta \widehat{\boldsymbol{d}}+\boldsymbol{f}(\boldsymbol{d})-\Delta \widetilde{\boldsymbol{d}})
$$

hence, the first term on the right hand side of (14) can be written as

$$
\left(\nabla_{d} \boldsymbol{f}(\boldsymbol{d})(\boldsymbol{u} \cdot \nabla) \mathbf{d}, \Delta \widehat{\boldsymbol{d}}-\boldsymbol{f}(\boldsymbol{d})\right)-\left(\nabla_{d} \boldsymbol{f}(\boldsymbol{d})(\Delta \widehat{\boldsymbol{d}}-\boldsymbol{f}(\boldsymbol{d})), \Delta \widehat{\boldsymbol{d}}-\boldsymbol{f}(\boldsymbol{d})\right)-\left(\nabla_{d} \boldsymbol{f}(\boldsymbol{d}) \Delta \widetilde{\boldsymbol{d}}, \Delta \widehat{\boldsymbol{d}}-\boldsymbol{f}(\boldsymbol{d})\right) .
$$

Taking into account that $\left\|\nabla_{d} \boldsymbol{f}(\boldsymbol{d})\right\|_{L^{\infty}\left(L^{\infty}\right)} \leq C$, weak estimates $\left(|\boldsymbol{u}|_{2} \leq C,\|\boldsymbol{d}\|_{1} \leq C\right)$ and the strong regularity for $\widetilde{\boldsymbol{d}} \in L^{\infty}\left(0,+\infty ; \boldsymbol{H}^{3}(\Omega)\right)$, we can bound these terms by:

$$
\begin{aligned}
& C\left(|\boldsymbol{u}|_{\infty}|\nabla \boldsymbol{d}|_{2}|\Delta \widehat{\boldsymbol{d}}-\boldsymbol{f}(\boldsymbol{d})|+|\Delta \widehat{\boldsymbol{d}}-\boldsymbol{f}(\boldsymbol{d})|_{2}^{2}+|\Delta \widetilde{\boldsymbol{d}}|_{2}|\Delta \widehat{\boldsymbol{d}}-\boldsymbol{f}(\boldsymbol{d})|_{2}\right) \\
& \leq C\left(\|\boldsymbol{u}\|_{1}^{1 / 2}\|\boldsymbol{u}\|_{2}^{1 / 2}\|\mathbf{d}\|_{1}|\Delta \widehat{\boldsymbol{d}}-\boldsymbol{f}(\boldsymbol{d})|_{2}+|\Delta \widehat{\boldsymbol{d}}-\boldsymbol{f}(\boldsymbol{d})|_{2}^{2}+1\right) \\
& \leq \frac{\nu}{18}\|\boldsymbol{u}\|_{2}^{2}+\frac{C}{\nu}|\Delta \widehat{\boldsymbol{d}}-\boldsymbol{f}(\boldsymbol{d})|_{2}^{2}+C\left(|\Delta \widehat{\boldsymbol{d}}-\boldsymbol{f}(\boldsymbol{d})|_{2}^{2}+1\right) .
\end{aligned}
$$


The second term on the right hand side of (14) is estimated by

$$
\begin{aligned}
& |-((\nabla \boldsymbol{u} \cdot \nabla) \boldsymbol{d}, \nabla(\Delta \widehat{\boldsymbol{d}}-\boldsymbol{f}(\boldsymbol{d})))| \leq C|\nabla \boldsymbol{u}|_{6}|\nabla \boldsymbol{d}|_{3}|\nabla(\Delta \widehat{\boldsymbol{d}}-\boldsymbol{f}(\boldsymbol{d}))|_{2} \\
& \leq \frac{\nu}{18}\|\boldsymbol{u}\|_{2}^{2}+\frac{C}{\nu}\|\boldsymbol{d}\|_{1}\|\boldsymbol{d}\|_{2}|\nabla(\Delta \widehat{\boldsymbol{d}}-\boldsymbol{f}(\boldsymbol{d}))|_{2}^{2} \\
& \leq \frac{\nu}{18}\|\boldsymbol{u}\|_{2}^{2}+\frac{C}{\nu}\left(1+|\Delta \widehat{\boldsymbol{d}}-\boldsymbol{f}(\boldsymbol{d})|_{2}\right)|\nabla(\Delta \widehat{\boldsymbol{d}}-\boldsymbol{f}(\boldsymbol{d}))|_{2}^{2} .
\end{aligned}
$$

Analogously, the last term on the right hand side of (14) is bounded by

$$
\begin{aligned}
& \left|\left(\boldsymbol{u} \cdot \nabla^{2} \mathbf{d}, \nabla(\Delta \widehat{\boldsymbol{d}}-\boldsymbol{f}(\boldsymbol{d}))\right)\right| \leq C|\boldsymbol{u}|_{\infty}\left|\nabla^{2} \boldsymbol{d}\right|_{2}|\nabla(\Delta \widehat{\boldsymbol{d}}-\boldsymbol{f}(\boldsymbol{d}))|_{2} \\
& \leq \frac{\nu}{18}\|\boldsymbol{u}\|_{2}^{2}+\frac{C}{\nu}\|\boldsymbol{d}\|_{2}^{2}|\nabla(\Delta \widehat{\boldsymbol{d}}-\boldsymbol{f}(\boldsymbol{d}))|_{2}^{2} \\
& \leq \frac{\nu}{18}\|\boldsymbol{u}\|_{2}^{2}+\frac{C}{\nu}\left(1+|\Delta \widehat{\boldsymbol{d}}-\boldsymbol{f}(\boldsymbol{d})|_{2}^{2}\right)|\nabla(\Delta \widehat{\boldsymbol{d}}-\boldsymbol{f}(\boldsymbol{d}))|_{2}^{2} .
\end{aligned}
$$

Consequently, applying previous estimates in (14) and considering that $C / \nu$ is uniformly bounded respect to $\nu$ as $\nu \geq \nu_{0}$, we arrive at

$$
\begin{aligned}
& \frac{d}{d t}|\Delta \widehat{\boldsymbol{d}}-\boldsymbol{f}(\boldsymbol{d})|_{2}^{2}+2|\nabla(\Delta \widehat{\boldsymbol{d}}-\boldsymbol{f}(\boldsymbol{d}))|_{2}^{2} \leq \frac{\nu}{3}\|\boldsymbol{u}\|_{2}^{2}+C\left(1+|\Delta \widehat{\boldsymbol{d}}-\boldsymbol{f}(\boldsymbol{d})|_{2}^{2}\right) \\
& +\frac{C}{\nu}\left(1+|\Delta \widehat{\boldsymbol{d}}-\boldsymbol{f}(\boldsymbol{d})|_{2}^{2}\right)|\nabla(\Delta \widehat{\boldsymbol{d}}-\boldsymbol{f}(\boldsymbol{d}))|_{2}^{2} .
\end{aligned}
$$

From (13) and (15) we obtain (12).

\section{Global solution of the initial-value problem}

Definition 5 We say that $(\boldsymbol{u}, \boldsymbol{d})$ is a weak solution of (1)-(3) if

$$
\begin{gathered}
\nabla \cdot \boldsymbol{u}=0 \text { in } Q,\left.\quad \boldsymbol{u}\right|_{\Sigma}=0,\left.\quad \boldsymbol{d}\right|_{\Sigma}=\boldsymbol{h}, \\
\|(\boldsymbol{u}(t), \boldsymbol{d}(t))\|_{0 \times 1} \leq C_{1} \quad \forall t \geq 0 \quad \text { i.e. }(\boldsymbol{u}, \boldsymbol{d}) \in L^{\infty}\left(0,+\infty ; \boldsymbol{L}^{2} \times \boldsymbol{H}^{1}\right), \\
\forall \gamma>0, \quad e^{-\gamma t} \int_{0}^{t} e^{\gamma s}\|(\boldsymbol{u}(s), \boldsymbol{d}(s))\|_{1 \times 2}^{2} d s \leq C_{2}, \quad \forall t \geq 0,
\end{gathered}
$$

verifying

$$
\begin{aligned}
& \left\langle\partial_{t} \boldsymbol{u}, \boldsymbol{v}\right\rangle+((\boldsymbol{u} \cdot \nabla) \boldsymbol{u}, \boldsymbol{v})+(\nabla \boldsymbol{u}, \nabla \boldsymbol{v})+\left(\nabla \boldsymbol{d}^{t} \Delta \boldsymbol{d}, \boldsymbol{v}\right)=0 \quad \forall \boldsymbol{v} \in \boldsymbol{V}, \\
& \partial_{t} \boldsymbol{d}+(\boldsymbol{u} \cdot \nabla) \boldsymbol{d}+\boldsymbol{f}(\boldsymbol{d})-\Delta \boldsymbol{d}=0, \quad|\boldsymbol{d}| \leq 1 \quad \text { a.e. in } Q \\
& \boldsymbol{u}(0)=\boldsymbol{u}_{0}, \quad \boldsymbol{d}(0)=\boldsymbol{d}_{0} \quad \text { in } \Omega .
\end{aligned}
$$

In the finite time case $(T<\infty)$, (17) holds even when $\gamma=0$, i.e. $(\boldsymbol{u}, \boldsymbol{d}) \in L^{2}\left(0, T ; \boldsymbol{H}^{1} \times \boldsymbol{H}^{2}\right)$.

Remark: (16) and (17) imply that $\left(\partial_{t} \boldsymbol{u}, \partial_{t} \boldsymbol{d}\right) \in L_{l o c}^{4 / 3}\left([0, \infty) ; \boldsymbol{V}^{\boldsymbol{\prime}} \times \boldsymbol{L}^{2}\right)$.

Definition 6 We say that $(\boldsymbol{u}, p, \boldsymbol{d})$ a weak solution of (1)-(3) is also a strong solution if

$$
\|(\boldsymbol{u}(t), \boldsymbol{d}(t))\|_{1 \times 2} \leq C_{3} \quad \forall t \geq 0,
$$




$$
\forall \gamma>0, \quad e^{-\gamma t} \int_{0}^{t} e^{\gamma s}\|(\boldsymbol{u}(s), \boldsymbol{d}(s))\|_{2 \times 3}^{2} d s \leq C_{4}, \quad \forall t \geq 0,
$$

verifying the following system a.e. in $Q$ :

$$
\left\{\begin{array}{l}
\partial_{t} \boldsymbol{u}+(\boldsymbol{u} \cdot \nabla) \boldsymbol{u}-\nu \Delta \boldsymbol{u}+\nabla p=-\nabla \boldsymbol{d}^{t} \Delta \boldsymbol{d}, \quad \nabla \cdot \boldsymbol{u}=0 \\
\partial_{t} \boldsymbol{d}+(\boldsymbol{u} \cdot \nabla) \boldsymbol{d}=\Delta \boldsymbol{d}-\boldsymbol{f}(\boldsymbol{d}), \quad|\boldsymbol{d}| \leq 1
\end{array}\right.
$$

In the finite time case $(T<\infty)$, again $\gamma=0$ can be taken in (19).

Remark: (18) and (19) imply that for all $\gamma>0$ and for all $t \geq 0$ :

$$
\begin{gathered}
e^{-\gamma t} \int_{0}^{t} e^{\gamma s}\left|\partial_{t} \boldsymbol{u}(s)\right|_{2}^{2} d s \leq C_{5}, \\
\left|\partial_{t} \boldsymbol{d}(t)\right|_{2} \leq C_{6}, \quad e^{-\gamma t} \int_{0}^{t} e^{\gamma s}\left\|\partial_{t} \boldsymbol{d}(s)\right\|_{1}^{2} d s \leq C_{7},
\end{gathered}
$$

and

$$
e^{-\gamma t} \int_{0}^{t} e^{\gamma s}|\nabla p(s)|_{2}^{2} d s \leq C_{8} .
$$

\section{Theorem 7 (Existence and uniqueness of the initial-valued problem)}

(1) Let $\Omega$ be a bounded domain in $\mathbb{R}^{3}$ with boundary $\partial \Omega$ of class $C^{1,1}$. Assume $\left(\boldsymbol{u}_{0}, \boldsymbol{d}_{0}\right) \in$ $\boldsymbol{H} \times \boldsymbol{H}^{1}$ with $\left|\boldsymbol{d}_{0}\right| \leq 1$ in $\Omega, \boldsymbol{h} \in L^{\infty}\left(0,+\infty ; H^{3 / 2}(\partial \Omega)\right)$ with $|\boldsymbol{h}| \leq 1$ on $\Sigma$ and $\partial_{t} \boldsymbol{h} \in$ $L^{\infty}\left(0,+\infty ; L^{2}(\partial \Omega)\right)$, verifying the compatibility condition $\left.\boldsymbol{d}_{0}\right|_{\partial \Omega}=\boldsymbol{h}(0)$. Then there exists a weak solution $(\boldsymbol{u}, \boldsymbol{d})$ of (1)-(3) in $[0,+\infty)$ which verifies (16)-(17) with constants $C_{1}, C_{2}$ independent of $\nu$ for each $\nu \geq 1 / 2$, and the follow energy inequality:

$$
\begin{aligned}
& |\boldsymbol{u}(t)|_{2}^{2}+|\nabla \widehat{\boldsymbol{d}}(t)|_{2}^{2}+2 \int_{0}^{t}\left(\nu|\nabla \boldsymbol{u}|_{2}^{2}+|\Delta \widehat{\boldsymbol{d}}|_{2}^{2}\right) \\
\leq & \left|\boldsymbol{u}_{0}\right|_{2}^{2}+2 \int_{0}^{t} \int_{\Omega}\left(f(\boldsymbol{d}) \cdot \Delta \widehat{\boldsymbol{d}}-(\boldsymbol{u} \cdot \nabla) \boldsymbol{d} \cdot \partial_{t} \widetilde{\boldsymbol{d}}\right)
\end{aligned}
$$

where the lifting function $\widetilde{\boldsymbol{d}}$ is defined as in Section 3.

(2) If moreover, $\partial \Omega$ is of class $C^{2,1},\left(\boldsymbol{u}_{0}, \boldsymbol{d}_{0}\right) \in \boldsymbol{H}^{1} \times \boldsymbol{H}^{2}$ with $\left\|\left(\boldsymbol{u}_{0}, \boldsymbol{d}_{0}\right)\right\|_{H^{1} \times H^{2}} \leq M_{0}, \boldsymbol{h} \in$ $L^{\infty}\left(0,+\infty ; H^{5 / 2}(\partial \Omega)\right)$ and $\partial_{t} \boldsymbol{h} \in L^{\infty}\left(0,+\infty ; H^{1 / 2}(\partial \Omega)\right)$, for each $\nu \geq \nu_{0}$, with $\nu_{0}=\nu_{0}\left(M_{0}, \boldsymbol{h}, \partial_{t} \boldsymbol{h}\right)$, there exists an unique strong solution of (1)-(3) in $[0,+\infty)$, which verifies (18) and (19) with constants $C_{3}, C_{4}$ independent of $\nu$.

(3) If $\left(\boldsymbol{u}_{1}, \boldsymbol{d}_{1}\right)$ is a weak solution of (1)-(3) which verifies the energy inequality (24) and $\left(\boldsymbol{u}_{2}, \boldsymbol{d}_{2}\right)$ is a strong solution of (1)-(3), then both solutions coincide.

Proof:

(1) In the proof of this part a semi-Galerkin method will be used. Let $\left\{\boldsymbol{w}_{i}\right\}_{n} \geq 1$ a "special" basis of $\boldsymbol{V}$ formed by eigenfunctions of the Stokes problem

$$
\left(\nabla \boldsymbol{w}_{i}, \nabla \mathbf{v}\right)=\lambda_{i}\left(\boldsymbol{w}_{i}, \mathbf{v}\right) \quad \forall \mathbf{v} \in \boldsymbol{V}, \boldsymbol{w}_{i} \in \boldsymbol{V}, \quad \text { with }\left\|\boldsymbol{w}_{i}\right\|_{L^{2}}=1, \quad \lambda_{i} \nearrow+\infty .
$$

Let $\boldsymbol{V}^{m}$ be the finite-dimensional subspace spanned by $\left\{\boldsymbol{w}_{1}, \boldsymbol{w}_{2}, \ldots, \boldsymbol{w}_{n}\right\}$. 
For each $m \geq 1$, we say that $\left(\boldsymbol{u}_{m}, \boldsymbol{d}_{m}\right)$ is an approximate solution, if $\boldsymbol{u}_{m}:[0,+\infty) \mapsto \boldsymbol{V}^{m}$ and $\boldsymbol{d}_{m}:[0,+\infty) \mapsto \boldsymbol{H}^{2}$ with $\widehat{\boldsymbol{d}}_{m}=\boldsymbol{d}_{m}-\tilde{\boldsymbol{d}}$ and $\widetilde{\boldsymbol{d}}$ the lifting function given in Section 3 (in particular, from regularity hypothesis of $\boldsymbol{h}$, one has that $\widetilde{\boldsymbol{d}} \in L^{\infty}\left(\boldsymbol{H}^{2}\right)$ and $\left.\widetilde{\boldsymbol{d}}_{t} \in L^{\infty}\left(\boldsymbol{L}^{2}\right)\right)$, and the following variational formulation holds:

$$
\left\{\begin{array}{l}
\left(\partial_{t} \boldsymbol{u}_{m}(t), \boldsymbol{v}_{m}\right)+\left(\left(\boldsymbol{u}_{m}(t) \cdot \nabla\right) \boldsymbol{u}_{m}(t), \boldsymbol{v}_{m}\right)+\nu\left(\nabla \boldsymbol{u}_{m}(t), \nabla \boldsymbol{v}_{m}\right) \\
+\left(\nabla \boldsymbol{d}_{m}^{t}(t) \Delta \boldsymbol{d}_{m}(t), \boldsymbol{v}_{m}\right)=0 \quad \forall \boldsymbol{v}_{m} \in \boldsymbol{V}^{m}, \quad \text { a.e. in } t, \\
\partial_{t} \widehat{\boldsymbol{d}}_{m}(t)+\left(\boldsymbol{u}_{m}(t) \cdot \nabla\right) \boldsymbol{d}_{m}(t)=\Delta \widehat{\boldsymbol{d}}_{m}(t)-\boldsymbol{f}\left(\boldsymbol{d}_{m}(t)\right), \quad\left|\boldsymbol{d}_{m}\right| \leq 1, \quad \text { a.e. in } Q, \\
\boldsymbol{u}_{m}(0)=\boldsymbol{u}_{0 m}=P_{m}\left(\boldsymbol{u}_{0}\right), \quad \boldsymbol{d}_{m}(0)=\boldsymbol{d}_{0} \quad \text { in } \Omega .
\end{array}\right.
$$

Here, $P_{m}: \boldsymbol{H} \mapsto \boldsymbol{V}^{m}$ denotes the usual orthogonal projector from $\boldsymbol{H}$ onto $\boldsymbol{V}^{m}$. In particular, $\boldsymbol{u}_{0 m} \rightarrow \boldsymbol{u}_{0}$ in $\boldsymbol{L}^{2}$.

The existence and uniqueness of local in time solution of (25) (in $Q_{T}$, for small enough $T$ ) is proved in the Appendix. Moreover, one has the estimates (independent of $m$ ): $\boldsymbol{u}_{m}$ bounded in $L^{\infty}(0, T ; \boldsymbol{H}) \cap L^{2}(0, T ; \boldsymbol{V})$ and $\boldsymbol{d}_{m}$ bounded in $L^{\infty}\left(0, T ; \boldsymbol{H}^{1}\right) \cap L^{2}\left(0, T ; \boldsymbol{H}^{2}\right)$. This suffices to control nonlinear terms and to pass to the limit in (25). Therefore, we get a weak solution of initial-valued problem (1)-(3) in $[0, T]$. Next, to extend the solution to whole $[0,+\infty)$ we will prove that the approximate solutions $\left(\boldsymbol{u}_{m}(t), \boldsymbol{d}_{m}(t)\right)$ are bounded in $[0,+\infty)$. By using the lifting (5) (Section 2 ), the approximate problem (25) can be rewritten as follows:

$$
\left\{\begin{array}{c}
\left(\partial_{t} \boldsymbol{u}_{m}(t), \boldsymbol{v}_{m}\right)+\left(\left(\boldsymbol{u}_{m}(t) \cdot \nabla\right) \boldsymbol{u}_{m}(t), \boldsymbol{v}_{m}\right)+\nu\left(\nabla \boldsymbol{u}_{m}(t), \nabla \boldsymbol{v}_{m}\right) \\
+\left(\nabla \boldsymbol{d}_{m}^{t}(t) \Delta \boldsymbol{d}_{m}(t), \boldsymbol{v}_{m}\right)=0 \quad \forall \boldsymbol{v}_{m} \in \boldsymbol{V}^{m}, \quad \text { a.e. } t, \\
\partial_{t} \widehat{\boldsymbol{d}}_{m}(t)+\left(\boldsymbol{u}_{m}(t) \cdot \nabla\right) \boldsymbol{d}_{m}(t)=\Delta \boldsymbol{d}_{m}(t)-\boldsymbol{f}\left(\boldsymbol{d}_{m}(t)\right)-\partial_{t} \widetilde{\boldsymbol{d}}(t) \quad \text { in } Q, \\
\boldsymbol{u}_{m}(0)=\boldsymbol{u}_{0 m}=P_{m}\left(\boldsymbol{u}_{0}\right), \quad \boldsymbol{d}_{m}(0)=\boldsymbol{d}_{0} \quad \text { in } \Omega .
\end{array}\right.
$$

Notice that $\widehat{\boldsymbol{d}}_{m}$ and $\widetilde{\boldsymbol{d}}$ are not the same functions in (25) and (26) respectively, since the lifting functions furnished in (5) or (10) are different, but the function $\boldsymbol{d}_{m}$ does not change.

From (7), one has in particular

$$
\frac{d}{d t}\left(\left|\boldsymbol{u}_{m}\right|_{2}^{2}+\left|\nabla \widehat{\boldsymbol{d}}_{m}\right|_{2}^{2}\right)+C_{0}\left(\left|\boldsymbol{u}_{m}\right|_{2}^{2}+\left|\nabla \widehat{\boldsymbol{d}}_{m}\right|_{2}^{2}\right) \leq 2\left(\left|\boldsymbol{f}\left(\boldsymbol{d}_{m}\right)\right|_{2}^{2}+\left|\partial_{t} \widetilde{\boldsymbol{d}}\right|_{2}^{2}\right) \leq C,
$$

where $C_{0}=\min \left\{\frac{2 \nu}{P}, \frac{1}{P}\right\}$ and $P$ is a Poincaré constant (for each $\nu \geq 1 / 2, C_{0}=1 / P$ a constant independent of $\nu)$. In the last estimate we have used that $\left|\boldsymbol{f}\left(\boldsymbol{d}_{m}\right)\right|_{2}^{2}$ is bounded in $L^{\infty}(0,+\infty)$ and $\left|\partial_{t} \widetilde{\boldsymbol{d}}\right|_{2}^{2} \in L^{\infty}(0,+\infty)$. Multiplying by $e^{C_{0} t}$,

$$
\frac{d}{d t}\left(e^{C_{0} t}\left(\left|\boldsymbol{u}_{m}\right|_{2}^{2}+\left|\nabla \widehat{\boldsymbol{d}}_{m}\right|_{2}^{2}\right)\right) \leq C e^{C_{0} t}
$$

and integrating in $[0, t]$ we have

$$
\left|\boldsymbol{u}_{m}(t)\right|_{2}^{2}+\left|\nabla \widehat{\boldsymbol{d}}_{m}(t)\right|_{2}^{2} \leq e^{-C_{0} t}\left(\left|\boldsymbol{u}_{0 m}\right|_{2}^{2}+\left|\nabla \widehat{\boldsymbol{d}}_{0}\right|_{2}^{2}\right)+C\left(1-e^{C_{0} t}\right) \leq\left|\boldsymbol{u}_{0}\right|_{2}^{2}+\left|\nabla \widehat{\boldsymbol{d}}_{0}\right|_{2}^{2}+C
$$

for all $t \geq 0$, with $C>0$ a constant independent of $\nu$, hence (16) holds with a constant $C_{1}$ independent of $\nu$ for all $\nu \geq 1 / 2$. 
Now, getting back to (7), multiplying by $e^{\gamma t}$ for any $\gamma>0$ and using the uniform in time estimates (28), we get

$$
\frac{d}{d t}\left(e^{\gamma t}\left(\left|\boldsymbol{u}_{m}\right|_{2}^{2}+\left|\widehat{\boldsymbol{d}}_{m}\right|_{2}^{2}\right)\right)+e^{\gamma t} C_{0}\left(\left|\nabla \boldsymbol{u}_{m}\right|_{2}^{2}+\left|\Delta \widehat{\boldsymbol{d}}_{m}\right|_{2}^{2}\right) \leq C e^{\gamma t}
$$

From this last differential inequality is easy to deduce (17) for a constant $C_{2}$ independent of $\nu$ for all $\nu \geq 1 / 2$. Then, existence of weak solution of (1)-(3) in $(0,+\infty)$ can be proved by means of a rather standard pass to the limit argument.

To obtain (24), we consider the approximated problem in the formulation (25). By taking $\boldsymbol{v}_{m}=\boldsymbol{u}_{m}$ as test function, multiplying the $\boldsymbol{d}_{m}$-system by $-\Delta \widehat{\boldsymbol{d}}_{m}$, adding up and taking into account that $\left(\nabla \boldsymbol{d}_{m}^{t} \Delta \widehat{\boldsymbol{d}}_{m}, \boldsymbol{u}_{m}\right)=\left(\left(\boldsymbol{u}_{m} \cdot \nabla\right) \boldsymbol{d}_{m}, \Delta \boldsymbol{d}_{m}\right)-\left(\left(\boldsymbol{u}_{m} \cdot \nabla\right) \boldsymbol{d}_{m}, \partial_{t} \widetilde{\boldsymbol{d}}_{m}\right)$ (notice that now, with the lifting function given in (10), $\Delta \boldsymbol{d} \neq \Delta \widehat{\boldsymbol{d}}$ in general) and taking limit as $m$ goes to $\infty$, one arrives at (24).

(2) Let $\left(\boldsymbol{u}_{m}, \boldsymbol{d}_{m}\right)$ be the solution in $(0, \infty)$ of problem (25), obtained in the previous section. Now, the lifting function is more regular, concretely $\widetilde{\boldsymbol{d}} \in L^{\infty}\left(\boldsymbol{H}^{3}\right)$ and $\widetilde{\boldsymbol{d}}_{t} \in L^{\infty}\left(\boldsymbol{H}^{1}\right)$. If we denote

$$
\Phi_{1}(t)=\left\|\boldsymbol{u}_{m}\right\|_{1}^{2}, \quad \Phi_{2}(t)=\left|\Delta \widehat{\boldsymbol{d}}_{m}-\boldsymbol{f}\left(\boldsymbol{d}_{m}\right)\right|_{2}^{2}, \quad \Psi_{1}(t)=\left\|\boldsymbol{u}_{m}\right\|_{2}^{2}, \quad \Psi_{2}(t)=\left|\nabla\left(\Delta \widehat{\boldsymbol{d}}_{m}-\boldsymbol{f}\left(\boldsymbol{d}_{m}\right)\right)\right|_{2}^{2}
$$

and

$$
\Phi=\Phi_{1}+\Phi_{2}
$$

then (12) is rewritten as follows:

$$
\Phi^{\prime}+\left(\nu-\frac{E}{\nu} \Phi_{1}^{1 / 2}\right) \Psi_{1}+\left(2-\frac{E}{\nu}\left(1+\Phi_{2}\right)\right) \Psi_{2} \leq D(1+\Phi), \quad \text { a.e. } t \in[0, \infty),
$$

where $E, D>0$ are constants (independent of $\nu$ ).

We will prove that $\Phi(t) \leq M$ for all $t \in[0, \infty)$, where $M$ will be a constant independent of $\nu$ that we will specify latter. By an absurd argument, let $t^{*}>0$ be a time such that

$$
\Phi\left(t^{*}\right)=M \quad \text { and } \quad \Phi(s)<M \quad \forall t \in\left[0, t^{*}\right) .
$$

Next, we shall assume that $\nu \geq \nu_{0}$ where $\nu_{0}=\nu_{0}(E, M)$ is a constant verifying

$$
\nu_{0}-\frac{E}{\nu_{0}} M^{1 / 2} \geq 1 \quad \text { and } \quad 2-\frac{E}{\nu_{0}}(1+M) \geq 1
$$

(recall that $E$ is independent of $\nu$ ). In particular $\nu_{0}>1$. Then,

$$
\Phi^{\prime}+\Psi \leq D(1+\Phi) \quad \text { in }\left[0, t^{*}\right]
$$

We denote $P=1 / \bar{C}$ where $\bar{C}$ is the Poincaré constant such that $\Phi \leq \bar{C} \Psi$. Therefore, $\Psi \geq P \Phi$, hence

$$
\Phi^{\prime}+P \Phi \leq D(1+\Phi) \quad \text { in }\left[0, t^{*}\right] .
$$

Multiplying (31) by $e^{P t}$ and integrating in $\left[0, t^{*}\right]$ one finds:

$$
\Phi\left(t^{*}\right) \leq \Phi(0) e^{-P t^{*}}+D e^{-P t^{*}} \int_{0}^{t^{*}} e^{P s}(1+\Phi(s)) d s .
$$


By using estimate (17), one has $\Phi\left(t^{*}\right) \leq \Phi(0)+\frac{D}{P}\left(1-e^{-P t^{*}}\right)+D C_{2}$. Since $\nu_{0}>1, \forall \nu \geq$ $\nu_{0}$, the constant $C_{2}$ is independent of $\nu$. Therefore, if we choose a constant $M>0$ such that $\Phi(0)+\frac{D}{P}+D C_{2}<M$ (notice that $M$ is independent of $\nu$ ), we arrive at contradiction.

Consequently, if $\nu \geq \nu_{0}, \Phi(t)=\left\|\boldsymbol{u}_{m}\right\|_{1}^{2}+\left|\Delta \widehat{\boldsymbol{d}}_{m}-\boldsymbol{f}\left(\boldsymbol{d}_{m}\right)\right|_{2}^{2}$ is bounded for any $t \in[0, \infty]$. Finally, taking into account Lemma 3 a), (18) is deduced. Getting back to (30) we have

$$
\Phi^{\prime}+\Psi \leq D(1+\Phi) \quad \text { in }[0, \infty) .
$$

Multiplying by $e^{\gamma t}$ for any $\gamma>0$ and integrating in $[0, t]$ it is easy to deduce that

$$
e^{-\gamma t} \int_{0}^{t} e^{\gamma s} \Psi(s) d s \leq C \quad \forall t \geq 0
$$

hence, (19) is deduced taking into account Lemma $3 \mathrm{~b}$ ). Finally, by passing to the limit, one finds that the limit $(\boldsymbol{u}, p, \boldsymbol{d})$ is the strong solution of (1)-(3) in $(0,+\infty)$.

Now, we are going to prove estimates (21), (22) and (23) using the semi-Galerkin problem (25). To prove (21) it suffices to consider $\partial_{t} \boldsymbol{u}_{m}$ as test function in the $\boldsymbol{u}_{m}$-system of (25), multiply by $e^{\gamma t}$, integrate in $[0, t]$ and use (18) and (19).

To prove (22), first one proves that $\left|\partial_{t} \widehat{\boldsymbol{d}}_{m}(t)\right|_{2}^{2}$ is bounded for all $t$. Indeed, from $\boldsymbol{d}_{m}$-system of (25) we have

$$
\left|\partial_{t} \widehat{\boldsymbol{d}}_{m}(t)\right|_{2}^{2} \leq C\left(\left\|\boldsymbol{u}_{m}\right\|_{1}^{2}\left\|\boldsymbol{d}_{m}\right\|_{2}^{2}+\left|\boldsymbol{f}\left(\boldsymbol{d}_{m}\right)-\Delta \boldsymbol{d}_{m}\right|_{2}^{2}+\left|\partial_{t} \widetilde{\boldsymbol{d}}\right|_{2}^{2}\right)
$$

and owing to (18) and (19) the right hand side of (32) is bounded. Second, differenciating with respect to the time the $\boldsymbol{d}_{m}$-system of (25) and multiplying by $\partial_{t} \widehat{\boldsymbol{d}}_{m}(t)$ one has

$$
\begin{aligned}
\frac{1}{2} \frac{d}{d t}\left|\partial_{t} \widehat{\boldsymbol{d}}_{m}\right|_{2}^{2}+\left|\partial_{t} \nabla \widehat{\boldsymbol{d}}_{m}\right|_{2}^{2} & =-\left(\left(\partial_{t} \boldsymbol{u}_{m} \cdot \nabla\right) \boldsymbol{d}_{m}, \partial_{t} \widehat{\boldsymbol{d}}_{m}\right)-\left(\left(\boldsymbol{u}_{m} \cdot \nabla\right) \partial_{t} \widetilde{\boldsymbol{d}}, \partial_{t} \widehat{\boldsymbol{d}}_{m}\right)-\left(\partial_{t} \boldsymbol{f}\left(\boldsymbol{d}_{m}\right), \partial_{t} \widehat{\boldsymbol{d}}_{m}\right) \\
& =\left(\left(\partial_{t} \boldsymbol{u}_{m} \cdot \nabla\right) \partial_{t} \widehat{\boldsymbol{d}}_{m}, \boldsymbol{d}_{m}\right)+\left(\left(\boldsymbol{u}_{m} \cdot \nabla\right) \partial_{t} \widehat{\boldsymbol{d}}_{m}, \partial_{t} \widetilde{\boldsymbol{d}}\right)-\left(\partial_{t} \boldsymbol{f}\left(\boldsymbol{d}_{m}\right), \partial_{t} \widehat{\boldsymbol{d}}_{m}\right)
\end{aligned}
$$

The three terms on the right hand side of the previous inequality are bounded respectively, by $\frac{1}{9}\left|\partial_{t} \nabla \widehat{\boldsymbol{d}}_{m}\right|_{2}^{2}+C\left|\partial_{t} \boldsymbol{u}_{m}\right|_{2}^{2}\left\|\boldsymbol{d}_{m}\right\|_{1}^{2}\left\|\boldsymbol{d}_{m}\right\|_{2}^{2}, \quad \frac{1}{9}\left|\partial_{t} \nabla \widehat{\boldsymbol{d}}_{m}\right|_{2}^{2}+C\left\|\boldsymbol{u}_{m}\right\|_{1}^{2}\left|\partial_{t} \widetilde{\boldsymbol{d}}\right|_{3}^{2} \quad$ and $\quad \frac{1}{9}\left|\partial_{t} \nabla \widehat{\boldsymbol{d}}_{m}\right|_{2}^{2}+C\left|\partial_{t} \widetilde{\boldsymbol{d}}\right|_{2}^{2}$, therefore, applying $L^{\infty}$ in time estimates already deduced, one arrives at

$$
\frac{d}{d t}\left|\partial_{t} \widehat{\boldsymbol{d}}_{m}\right|_{2}^{2}+\left|\partial_{t} \nabla \widehat{\boldsymbol{d}}_{m}\right|_{2}^{2} \leq C\left(1+\left|\partial_{t} \boldsymbol{u}_{m}\right|_{2}^{2}\right)
$$

Then, multiplying by $e^{\gamma t}$ for each $\gamma>0$, integrating in $[0, t]$ and using the bound (21) previously obtained, one arrives to (22).

Finally, estimate for pressure (23) can be deduced in a similar way from the $\boldsymbol{u}_{m}$-system.

(3) We will use an argument of strong/weak uniqueness (see for instance [9] for Navier Stokes case). Let $\left(\boldsymbol{u}^{1}, \boldsymbol{d}^{1}\right)$ be a weak solution of (1)-(3) verifying (24) and let $\left(\boldsymbol{u}^{2}, \boldsymbol{d}^{2}\right)$ be a regular solution. We denote $\boldsymbol{u}=\boldsymbol{u}^{1}-\boldsymbol{u}^{2}$ and $\boldsymbol{d}=\boldsymbol{d}^{1}-\boldsymbol{d}^{2}$ (notice that $\boldsymbol{d}=\widehat{\boldsymbol{d}}$ ). 
Adding the energy inequality (24) for $\left(\boldsymbol{u}^{1}, \boldsymbol{d}^{1}\right)$ and for $\left(\boldsymbol{u}^{2}, \boldsymbol{d}^{2}\right)$, and subtracting the result of multiplying the $\left(\boldsymbol{u}^{2}, \boldsymbol{d}^{2}\right)$-system by $\left(\boldsymbol{u}^{1},-\Delta \boldsymbol{d}^{1}\right)$ and the result of considering $\left(-\boldsymbol{u}^{2}, \Delta \widehat{\boldsymbol{d}}^{2}\right)$ as test function in $\left(\boldsymbol{u}^{1}, \boldsymbol{d}^{1}\right)$-system, and finally integrating in $\Omega \times[0, t]$ one arrives at:

$$
\begin{aligned}
& \frac{1}{2}\left(|\boldsymbol{u}(t)|_{2}^{2}+|\nabla \widehat{\boldsymbol{d}}(t)|_{2}^{2}\right)+\int_{0}^{t}\left(\nu|\nabla \boldsymbol{u}|_{2}^{2}+|\Delta \widehat{\boldsymbol{d}}|_{2}^{2}\right) \\
& \leq-\int_{0}^{t}\left((\boldsymbol{u} \cdot \nabla) \boldsymbol{u}^{2}, \boldsymbol{u}\right)-\int_{0}^{t}\left(\nabla \boldsymbol{d}^{t} \Delta \widehat{\boldsymbol{d}}^{2}, \boldsymbol{u}\right) \\
& +\int_{0}^{t}\left(\left(\boldsymbol{u}^{2} \cdot \nabla\right) \boldsymbol{d}, \Delta \widehat{\boldsymbol{d}}\right)-\int_{0}^{t}\left(\boldsymbol{f}\left(\boldsymbol{d}^{2}\right)-\boldsymbol{f}\left(\boldsymbol{d}^{1}\right), \Delta \widehat{\boldsymbol{d}}\right)-\int_{0}^{t}\left(\nabla \boldsymbol{d}^{t} \Delta \widehat{\boldsymbol{d}}, \boldsymbol{u}\right) .
\end{aligned}
$$

By bounding adequately the terms on the right hand side of (33) (see [2]), using that $f$ is locally lipschitz, $\left|\boldsymbol{d}^{1}\right| \leq 1,\left|\boldsymbol{d}^{2}\right| \leq 1$ and recalling that $\widehat{\boldsymbol{d}}=\boldsymbol{d}$, one arrives at

$$
\begin{aligned}
& |\boldsymbol{u}(t)|_{2}^{2}+|\nabla \boldsymbol{d}(t)|_{2}^{2}+\int_{0}^{t}\left(\nu|\nabla \boldsymbol{u}|_{2}^{2}+|\Delta \boldsymbol{d}|_{2}^{2}\right) \\
& \leq C \int_{0}^{t}\left(\left|\Delta \boldsymbol{d}^{2}\right|_{3}^{2}+\left|\boldsymbol{u}^{2}\right|_{4}^{8}+1\right)\left(|\boldsymbol{u}(s)|_{2}^{2}+|\nabla \boldsymbol{d}(s)|_{2}^{2}\right) .
\end{aligned}
$$

Since $\left(\boldsymbol{u}^{2}, \boldsymbol{d}^{2}\right)$ is a strong solution, $\left|\Delta \widehat{\boldsymbol{d}}^{2}\right|_{3}^{2}$ and $\left|\boldsymbol{u}^{2}\right|_{4}^{8}$ are bounded in $L^{1}(0, t)$. Applying Gronwall's Lemma, one has $\boldsymbol{u}=0$ and $\nabla \boldsymbol{d}=0$. Finally, since $\boldsymbol{d}=0$ on $\Sigma$, then $\boldsymbol{d}=0$. Therefore, uniqueness of strong/weak solutions for the initial-boundary problem (1)-(3) is proved.

\section{Solution of time-periodic problem}

In this section, we assume that $T>0$ is finite and fix.

Definition 8 We say that $(\boldsymbol{u}, p, \boldsymbol{d})$ is a regular time-periodic solution of (1), (2) and (4) if $\boldsymbol{u} \in$ $L^{2}\left(\boldsymbol{H}^{2}\right) \cap L^{\infty}\left(\boldsymbol{H}^{1}\right), \partial_{t} \boldsymbol{u} \in L^{2}\left(\boldsymbol{L}^{2}\right), p \in L^{2}\left(H^{1}\right), \boldsymbol{d} \in L^{2}\left(H^{3}\right) \cap L^{\infty}\left(H^{2}\right)$ and $\partial_{t} \boldsymbol{d} \in L^{2}\left(H^{1}\right) \cap L^{\infty}\left(L^{2}\right)$ satisfying (1) a.e. in $(0, T) \times \Omega$, boundary conditions (2) and periodic conditions $\boldsymbol{u}(0)=\boldsymbol{u}(T)$, $\boldsymbol{d}(0)=\boldsymbol{d}(T)$ in the sense of spaces $\boldsymbol{V}$ and $\boldsymbol{H}^{2}$ respectively.

Theorem 9 (Existence and uniqueness of time-periodic solutions) Let $\Omega$ be a bounded domain in $\mathbb{R}^{3}$ with boundary $\partial \Omega$ of class $C^{2,1}$ and $T>0$. If $\boldsymbol{h} \in L^{\infty}\left(0, T ; H^{5 / 2}(\partial \Omega)\right)$, $\partial_{t} \boldsymbol{h} \in$ $L^{\infty}\left(0, T ; H^{1 / 2}(\partial \Omega)\right)$ with $\boldsymbol{h}(0)=\boldsymbol{h}(T)$ and $\nu \geq \nu_{0}$, for a certain positive constant $\nu_{0}=\nu_{0}\left(T, \boldsymbol{h}_{t}\right)$, then there exists a regular periodic solution of (1), (2) and (4), which verifies (18) and (19) with constants $C_{3}$ and $C_{4}$ independent of $\nu$.

Proof: Let $(\boldsymbol{u}, \boldsymbol{d})$ a weak time-periodic solution in $(0, T)$ of problem (1), furnished as in [2] by means of a Galerkin method.

Here, we will consider $\widehat{\boldsymbol{d}}=\boldsymbol{d}-\widetilde{\boldsymbol{d}}$, where $\widetilde{\boldsymbol{d}}$ is the lifting function given in Section 2. From weak estimates of the weak time-periodic solutions made in [2], there exits a constant $C>0$ (independent of $\nu$ ) such that $\int_{0}^{T}\left(\nu|\nabla \boldsymbol{u}|_{2}^{2}+|\Delta \widehat{\boldsymbol{d}}|_{2}^{2}\right) d t \leq C$. Therefore, there is a $t_{0} \in[0, T]$ such that

$$
\nu\left|\nabla \boldsymbol{u}\left(t_{0}\right)\right|_{2}^{2}+\left|\Delta \widehat{\boldsymbol{d}}\left(t_{0}\right)\right|_{2}^{2} \leq \frac{C}{T}
$$


In particular, recalling (29) and that $|\boldsymbol{f}(\boldsymbol{d})| \leq \frac{1}{\varepsilon^{2}}$ one has that

$$
\Phi\left(t_{0}\right) \leq M_{0}, \quad \text { for all } \nu \geq 1
$$

( $\Phi$ is defined in (29)). We will prove that $\Phi(t)$ is bounded in the whole time interval $[0,+\infty)$ in two steps. For that, we will choose two constants $M_{1}$ and $M_{2}$ that we will specify latter.

First step: There are two positive constants $M_{1}=M_{1}\left(M_{0}\right)$ and $\nu_{1}=\nu_{1}\left(M_{0}\right)$, such that if $\nu \geq \nu_{1}$, then $\Phi(t) \leq M_{1}, \forall t \in\left[t_{0},+\infty\right]$.

We consider the strong solution of the problem (1), (2) with the initial condition fixed at $t_{0}:\left(\boldsymbol{u}\left(t_{0}\right), \boldsymbol{d}\left(t_{0}\right)\right)$, which is defined in $\left[t_{0},+\infty\right)$ provided $\nu \geq \nu_{1}\left(M_{0}\right)$ (see Theorem 7 ). Owing to uniqueness of weak/strong solution, this strong solution coincides in $\left[t_{0},+\infty\right)$ with the weak time-periodic solution. In particular, $\Phi(t) \leq M_{1}, \forall t \in\left[t_{0},+\infty\right)$ (indeed, it suffices to repeat the proof of Theorem 7 (2), starting from $t_{0}$ ).

Second step: There are two positive constants $M_{2}=M_{2}\left(M_{1}\right)$ and $\nu_{2}=\nu_{2}\left(M_{1}\right)$, such that if $\nu \geq \nu_{2}$, then $\Phi(t) \leq M_{2}, \forall t \in\left[0, t_{0}\right]$.

By time periodicity $\Phi(0)=\Phi(T)$, hence $\Phi(0) \leq M_{1}$. Again the strong solution of the problem (1), (2) with the initial condition fixed at $0:(\boldsymbol{u}(0), \boldsymbol{d}(0))$, is defined in $[0,+\infty)$ for each $\nu \geq \nu_{2}\left(M_{1}\right)$, verifies $\Phi(t) \leq M_{2}$ for all $t \geq 0$ and coincides with the weak time-periodic solution in $[0,+\infty)$.

Consequently, $\Phi^{\prime}+\Psi \leq D(1+\Phi)$ in $[0,+\infty)$, hence one has strong estimates (18) and (19) as in the proof of Theorem 7 (2).

Finally, the regularity for $\partial_{t} \boldsymbol{u}, \partial_{t} \boldsymbol{d}$ and $p$ is obtained as in the proof of Theorem 7 (2).

\section{Appendix}

Theorem 10 There is a time $T>0$ depending on $\boldsymbol{u}_{0}, \boldsymbol{d}_{0}, m$ and $\Omega$ such that the semi-Galerkin problem (25) has a unique solution in $Q_{T}$.

Proof: To prove the existence of semi-Galerkin approximate solution, a linearized argument will be used by splitting the problem (25) into the two following problems:

Let $T>0$ and $\overline{\boldsymbol{u}}^{m} \in \mathcal{C}\left([0, T] ; \boldsymbol{V}^{m}\right)$.

a) To find $\boldsymbol{d}^{m} \in L^{\infty}\left(0, T ; \boldsymbol{H}^{1}\right) \cap L^{2}\left(0, T ; \boldsymbol{H}^{2}\right)$ such that

$$
\left\{\begin{array}{l}
\partial_{t} \boldsymbol{d}^{m}+\left(\overline{\boldsymbol{u}}^{m} \cdot \nabla\right) \boldsymbol{d}^{m}=\Delta \boldsymbol{d}^{m}-\boldsymbol{f}\left(\boldsymbol{d}^{m}\right) \quad \text { in } Q_{T} \\
\boldsymbol{d}^{m}(0)=d_{0} \quad \text { in } \Omega, \quad \boldsymbol{d}^{m}=\boldsymbol{h} \quad \text { on } \Sigma_{T}
\end{array}\right.
$$

b) Known $\boldsymbol{d}^{m}$, to find $\boldsymbol{u}^{m} \in H^{1}\left(0, T ; \boldsymbol{V}^{m}\right)$ such that

$$
\left\{\begin{array}{l}
\left(\partial_{t} \boldsymbol{u}^{m}, \overline{\boldsymbol{u}}\right)+\left(\left(\overline{\boldsymbol{u}}^{m} \cdot \nabla\right) \boldsymbol{u}^{m}, \overline{\boldsymbol{u}}\right)+\left(\nabla \boldsymbol{u}^{m}, \nabla \overline{\boldsymbol{u}}\right)=-\left(\nabla \boldsymbol{d}^{m T} \Delta \boldsymbol{d}^{m}, \overline{\boldsymbol{u}}\right), \\
\forall \overline{\boldsymbol{u}} \in \boldsymbol{V}_{m}, \quad \text { a.e } t \in(0, T), \\
\boldsymbol{u}^{m}(0)=\boldsymbol{u}_{0}^{m} \quad \text { in } \Omega
\end{array}\right.
$$


First step: There exists an unique solution $\boldsymbol{d}^{m}$ of problem (35) which is bounded in $L^{\infty}\left(0, T ; \boldsymbol{H}^{1}\right) \cap$ $L^{2}\left(0, T ; \boldsymbol{H}^{2}\right)$ if $\overline{\boldsymbol{u}}^{m}$ is bounded in $C\left([0, T] ; \boldsymbol{L}^{2}(\Omega)\right)$.

It suffices to prove that if $\left\|\overline{\boldsymbol{u}}^{m}\right\|_{C\left([0, T] ; L^{2}(\Omega)\right)}^{2} \leq M$ (M>0 a constant), there exists a constant $C=C(m, M)$ such that $\left\|\boldsymbol{d}^{m}\right\|_{L^{\infty}\left(0, T ; H^{1}\right) \cap L^{2}\left(0, T ; H^{2}\right)} \leq C(m, M)$.

By considering the lifting function $\widetilde{\boldsymbol{d}}$ given in (10), the problem (35) can be rewrite as

$$
\left\{\begin{array}{l}
\partial_{t} \widehat{\boldsymbol{d}}^{m}+\left(\overline{\boldsymbol{u}}^{m} \cdot \nabla\right) \boldsymbol{d}^{m}=\Delta \widehat{\boldsymbol{d}}^{m}-f\left(\boldsymbol{d}^{m}\right) \quad \text { in } Q_{T} \\
\widehat{\boldsymbol{d}}^{m}(0)=0 \quad \text { in } \Omega, \quad \widehat{\boldsymbol{d}}^{m}=0 \quad \text { on } \Sigma_{T} .
\end{array}\right.
$$

Multiplying the previous system by $-\Delta \widehat{\boldsymbol{d}}^{m}$ and integrating in $\Omega$, one obtains:

$$
\frac{d}{d t}\left|\nabla \widehat{\boldsymbol{d}}^{m}\right|_{2}^{2}+\left|\Delta \widehat{\boldsymbol{d}}^{m}\right|_{2}^{2} \leq 2\left(\left|\overline{\boldsymbol{u}}^{m} \cdot \nabla \boldsymbol{d}^{m}\right|_{2}^{2}+\left|\boldsymbol{f}\left(\boldsymbol{d}^{m}\right)\right|_{2}^{2}\right)
$$

Since $\left|\boldsymbol{d}^{m}\right| \leq 1$ in $Q_{T}$, then $\left\|\boldsymbol{f}\left(\boldsymbol{d}^{m}\right)\right\|_{L^{\infty}\left(L^{2}\right)}^{2} \leq C$. On the other hand, by hypothesis $\left\|\overline{\boldsymbol{u}}^{m}\right\|_{L^{\infty}\left(L^{2}\right)}^{2} \leq$ $M$. In particular, since $\boldsymbol{u}^{m}(t) \in \boldsymbol{V}^{m}$, a finite dimensional space, one has $\left\|\overline{\boldsymbol{u}}^{m}\right\|_{L^{\infty}\left(L^{\infty}\right)}^{2} \leq C_{m} M$. Consequently,

$$
\left|\overline{\boldsymbol{u}}^{m} \cdot \nabla \boldsymbol{d}^{m}\right|_{2}^{2} \leq C_{m} M\left|\nabla \boldsymbol{d}^{m}\right|_{2}^{2} \leq C_{m} M\left(\left|\nabla \widehat{\boldsymbol{d}}^{m}\right|_{2}^{2}+|\nabla \widetilde{\boldsymbol{d}}|_{2}^{2}\right)
$$

Therefore,

$$
\frac{d}{d t}\left|\nabla \widehat{\boldsymbol{d}}^{m}\right|_{2}^{2}+\left|\Delta \widehat{\boldsymbol{d}}^{m}\right|_{2}^{2} \leq C_{m} M\left(\left|\nabla \widehat{\boldsymbol{d}}^{m}\right|_{2}^{2}+|\nabla \widetilde{\boldsymbol{d}}|_{2}^{2}\right)+C .
$$

By applying the Gronwall's Lemma, one obtains:

$$
\left|\nabla \widehat{\boldsymbol{d}}^{m}(t)\right|_{2}^{2}+\int_{0}^{t}\left|\Delta \widehat{\boldsymbol{d}}^{m}\right|_{2}^{2} d s \leq e^{C_{m} M T} \int_{0}^{t}\left(C_{m} M|\nabla \widetilde{\boldsymbol{d}}|_{2}^{2}+C\right) d s .
$$

Since $\widetilde{\boldsymbol{d}} \in L^{2}\left(0, T ; \boldsymbol{H}^{1}\right)$, the term on right hand side is bounded, hence $\boldsymbol{d}^{m}$ is bounded in $L^{\infty}\left(0, T ; \boldsymbol{H}^{1}\right) \cap$ $L^{2}\left(0, T ; \boldsymbol{H}^{2}\right)$ by a constant which depends on the bound of $\left\|\overline{\boldsymbol{u}}^{m}\right\|_{L^{\infty}\left(0, T ; L^{2}\right)}^{2}$ and on $m$. In particular,

$$
\left\|\nabla \widehat{\boldsymbol{d}}^{m}\right\|_{L^{\infty}\left(0, T ; L^{2}\right)}^{2} \leq e^{C_{m} M T} \int_{0}^{T}\left(C_{m} M|\nabla \widetilde{\boldsymbol{d}}|_{2}^{2}+C\right) d s:=K(T, M),
$$

where $K(T, M) \rightarrow 0$ as $T \rightarrow 0$, for each $M>0$. That finishes the first step.

Second step: There exists an unique solution $\boldsymbol{u}_{m}$ of problem (36) bounded in $H^{1}\left(0, T ; \boldsymbol{H}^{1}\right)$ by a constan which depends on the bound of $\left\|\overline{\boldsymbol{u}}^{m}\right\|_{L^{\infty}\left(0, T ; L^{2}\right)}$.

Assume that $\boldsymbol{u}^{m}=\sum_{i=1}^{m} \eta_{i}(t) \mathbf{w}^{i}$ then, the problem (36) can be written as the following first order differential system:

$$
\left\{\begin{array}{l}
\frac{d}{d t} \eta_{j}+\sum_{i=1}^{m}\left(\left(\overline{\boldsymbol{u}}^{m} \cdot \nabla\right) \boldsymbol{w}^{i}, \boldsymbol{w}^{j}\right) \eta_{i}+\eta_{j}=-\left(\nabla \boldsymbol{d}^{m T} \Delta \boldsymbol{d}^{m}, \boldsymbol{w}^{j}\right), \forall j=1, \ldots, m \\
\eta_{j}(0)=\left(\boldsymbol{u}_{0}, \boldsymbol{w}^{j}\right), \forall j=1, \ldots, m .
\end{array}\right.
$$

which has an unique solution defined in $\left[0, T_{m}\right)$ belonging to $H^{1}\left(0, T_{m}\right)^{m}$ for a certain $T_{m}>0$. Therefore, there exists a solution of $(36)$ in $\left[0, T_{m}\right)$, where

$$
T_{m}=T \quad \text { or } \quad \limsup _{t \rightarrow T_{m}}\left|\boldsymbol{u}^{m}(t)\right|_{2}=+\infty .
$$


We will prove that $T_{m}=T$. For that, we will see that $\boldsymbol{u}^{m}$ is bounded in $L^{\infty}\left(0, T_{m} ; \boldsymbol{L}^{2}(\Omega)\right) \cap$ $L^{2}\left(0, T_{m} ; \boldsymbol{H}^{1}(\Omega)\right)$. Indeed, by taking $\overline{\boldsymbol{u}}=\boldsymbol{u}^{m}$ in (36),

$$
\frac{1}{2} \frac{d}{d t}\left|\boldsymbol{u}^{m}\right|_{2}^{2}+\nu\left|\nabla \boldsymbol{u}^{m}\right|_{2}^{2}=-\int_{\Omega}\left(\nabla \boldsymbol{d}^{m t} \Delta \boldsymbol{d}^{m}\right) \cdot \boldsymbol{u}^{m}
$$

By using that $\nabla \cdot\left(\nabla \boldsymbol{d}^{m t} \nabla \boldsymbol{d}^{m}\right)=\nabla \boldsymbol{d}^{m t} \Delta \boldsymbol{d}^{m}+\nabla\left(\frac{\left|\nabla \boldsymbol{d}^{m}\right|^{2}}{2}\right)$ the previous term on right hand side is equal to $\int_{\Omega}\left(\nabla \boldsymbol{d}^{m t} \nabla \boldsymbol{d}^{m}\right) \nabla \boldsymbol{u}^{m} d x$.

Taking into account that $\left|\nabla \boldsymbol{u}^{m}(t)\right|_{\infty} \leq C_{m}\left|\nabla \boldsymbol{u}^{m}(t)\right|_{2}$ for any $t \in\left[0, T_{m}\right)$, one arrives to

$$
\frac{d}{d t}\left|\boldsymbol{u}^{m}\right|_{2}^{2}+\nu\left|\nabla \boldsymbol{u}^{m}\right|_{2}^{2} \leq \frac{C_{m}}{\nu}\left|\nabla \boldsymbol{d}^{m}\right|_{2}^{4}
$$

and integrating in $\left[0, T_{m}\right]$ one obtains

$$
\left|\boldsymbol{u}^{m}(t)\right|_{2}^{2}+\nu\left\|\nabla \boldsymbol{u}^{m}\right\|_{L^{2}\left(0, T_{m} ; L^{2}\right)}^{2} \leq\left|\boldsymbol{u}^{m}(0)\right|_{2}^{2}+\frac{C_{m}}{\nu} T_{m}\left\|\nabla \boldsymbol{d}^{m}\right\|_{L^{\infty}\left(0, T_{m} ; L^{2}\right)}^{4} \quad \forall t \in\left[0, T_{m}\right) .
$$

By using the first step, $\nabla \boldsymbol{d}^{m}$ is bounded in $L^{\infty}\left(0, T_{m} ; \boldsymbol{L}^{2}\right)$ if $\overline{\boldsymbol{u}}^{m}$ is bounded in $L^{\infty}\left(0, T_{m} ; \boldsymbol{L}^{2}\right)$. Therefore, the right hand side of (40) is bounded if $\overline{\boldsymbol{u}}^{m}$ is bounded in $L^{\infty}\left(0, T_{m} ; \boldsymbol{L}^{2}\right)$. Hence, $T_{m}=T$ and $\boldsymbol{u}^{m}$ is bounded in $L^{\infty}\left(0, T ; \boldsymbol{L}^{2}\right) \cap L^{2}\left(0, T ; \boldsymbol{H}^{1}\right)$ by a constant depending on the bound of $\left\|\overline{\boldsymbol{u}}^{m}\right\|_{L^{\infty}\left(0, T ; L^{2}\right)}$ and $m$. Finally, by applying this estimate in (39), one have that $\frac{d}{d t} \eta_{j}$ is bounded in $L^{2}(0, T)$, hence $\boldsymbol{u}^{m}$ is bounded in $H^{1}\left(0, T ; \boldsymbol{H}^{1}\right)$ by a constant depending on the bound of $\left\|\overline{\boldsymbol{u}}^{m}\right\|_{L^{\infty}\left(0, T ; L^{2}\right)}$ and $m$. Therefore second step is finished.

Third step: A fixed point argument implies the existence of solution of problem (25).

Let us define the operator

$$
\Phi_{T}: \overline{\boldsymbol{u}}^{m} \in C\left([0, T] ; \boldsymbol{V}_{m}\right) \rightarrow \boldsymbol{d}^{m} \in L^{2}\left(0, T ; \boldsymbol{H}^{2}\right) \cap L^{\infty}\left(0, T ; \boldsymbol{H}^{1}\right) \rightarrow \boldsymbol{u}^{m} \in H^{1}\left(0, T ; \boldsymbol{V}_{m}\right)
$$

where $\boldsymbol{d}^{m}$ is the solution of (35) in $[0, T]$ and $\boldsymbol{u}^{m}$ the solution of (36) in $[0, T] . \Phi_{T}$ is continuous because the solutions of (35) and (36) depend continuously on data.

Moreover, taking into account the previous two steps, given $\overline{\boldsymbol{u}}^{m} \in \mathcal{C}\left([0, T] ; \boldsymbol{V}_{m}\right)$ there exists an unique $\boldsymbol{u}^{m} \in H^{1}\left(0, T ; \boldsymbol{V}_{m}\right)$ solution of problem (36) bounded in $H^{1}\left(0, T ; \boldsymbol{V}_{m}\right)$ if $\overline{\boldsymbol{u}}^{m}$ is bounded in $\mathcal{C}\left([0, T] ; \boldsymbol{V}_{m}\right)$. Compactness of $H^{1}\left(0, T ; \boldsymbol{V}^{m}\right)$ into $\mathcal{C}\left([0, T] ; \boldsymbol{V}^{m}\right)$ (owing to $\boldsymbol{V}^{m}$ is a finite dimension space) gives that $\Phi_{T}$ is compact from $\mathcal{C}\left([0, T] ; \boldsymbol{V}_{m}\right)$ into itself.

On the other hand, we take $M>0$ such that $\left|\boldsymbol{u}_{0}\right|_{2}^{2} \leq M / 2$. If $\left|\overline{\boldsymbol{u}}^{m}(t)\right|_{2}^{2} \leq M$ for all $t \in[0, T]$, from (38) and (40) we have

$$
\left|\boldsymbol{u}^{m}(t)\right|_{2}^{2} \leq \frac{M}{2}+\frac{C}{\nu}\left(K(T, M)^{2}+\|\nabla \widetilde{\boldsymbol{d}}\|_{L^{\infty}\left(0, T ; L^{2}\right)}^{4}\right) .
$$

Then, since $K(T, M) \rightarrow 0$ as $T \rightarrow 0$, there exists $T_{0}>0$ small enough depending on $M$ such that $\left|\boldsymbol{u}^{m}(t)\right|_{2}^{2} \leq M, \forall t \in\left[0, T_{0}\right]$.

Therefore, applying Schauder's Theorem, there exists a fixed point $\boldsymbol{u}^{m} \in H^{1}\left(0, T_{0} ; \boldsymbol{V}_{m}\right)$. Moreover, $\boldsymbol{u}^{m}$ is bounded in $L^{\infty}\left(0, T_{0} ; \boldsymbol{L}^{2}(\Omega)\right) \cap L^{2}\left(0, T_{0} ; \boldsymbol{H}^{1}(\Omega)\right)$ and $\boldsymbol{d}^{m}$ is bounded in $L^{\infty}\left(0, T_{0} ; \boldsymbol{H}^{1}(\Omega)\right) \cap L^{2}\left(0, T_{0} ; \boldsymbol{H}^{2}(\Omega)\right)$ by constants depending on $M$. 


\section{References}

[1] F. Bethuel, H. Brezis, F. Hélein, Asymptotics for the minimization of a Ginzburg-Landau functional, Calc. Var. 1 (1993), 123-148.

[2] B. Climent-Ezquerra, F. Guillén-González, M.A. Rojas-Medar Reproductivity for a nematic liquid crystal model, Z. Angew. Math. Phys., 576 (2006) no. 6, 984-998.

[3] B. Climent-Ezquerra, F. Guillén-González, M.A. Rojas-Medar Regular time-reproductive solutions for generalized Boussinesq model with Neumann boundary conditions for temperature. Proc. R. Soc. A (2007) 463, 2153-2164.

[4] D. Coutand, S. Shkoller, Well posedness of the full Ericsen-Leslye model of nematic liquid crystals, Note C.R.A.S, t. 333, Serie I (2001), 919-924.

[5] F. Guillén-González, M.A. Rojas-Medar, M.A. Rodríguez-Bellido Sufficient conditions for regularity and uniqueness of a $3 D$ nematic liquid crystal model. Submitted.

[6] F.H. Lin Nonlinear theory of defects in nematic liquid crystals: phase transition and flow phenomena, Comm. Pure Appl. Math., 42 (1989), 789-814.

[7] F.H. Lin, C. Liu Non-parabolic dissipative systems modelling the flow of liquid crystals, Comm. Pure Appl. Math., 4 (1995), 501-537.

[8] F.H. Lin, C. Liu Existence of solutions for the Ericksen-Leslie system, Arch. Rat. Mech. Anal., 154 (2000), 135-156.

[9] P.L. Lions Mathematical Topics in Fluids Mechanics, Clarendon Press, Oxford, 1996. 